\title{
BMJ Health \& Care Informatics \\ Are child health information services a viable source of accurate vaccination data for clinicians working in paediatric emergency departments in England?
}

\author{
Rachel Isba (D) , ${ }^{1,2}$ Nigel Davies, ${ }^{3}$ Jo Knight (i) ${ }^{1}$
}

To cite: Isba R, Davies N, Knight J. Are child health information services a viable source of accurate vaccination data for clinicians working in paediatric emergency departments in England? BMJ Health Care Inform 2021;28:e100486. doi:10.1136/ bmjhci-2021-100486

Received 27 September 2021 Accepted 11 December 2021

D) Check for updates

(c) Author(s) (or their employer(s)) 2021. Re-use permitted under CC BY-NC. No commercial re-use. See rights and permissions. Published by BMJ.

${ }^{1}$ Lancaster Medical School, Lancaster University, Lancaster, UK

${ }^{2}$ Emergency Department, North Manchester General Hospital, Manchester University NHS Foundation Trust, Manchester, UK

${ }^{3}$ School of Computing and Communication, Lancaster University, Lancaster, UK

Correspondence to Professor Rachel Isba; rachel.isba@nhs.net

\section{ABSTRACT}

Vaccination is a global success story, yet UK coverage remains undertarget for a number of diseases. The paediatric emergency department (PED) offers the potential for opportunistic vaccination interventions. Objectives To map the Greater Manchester (GM) Child Health Information System network to see if it was a viable source of vaccination data for clinicians working in the PED as a case study.

Methods Postprimary care vaccination management systems for GM were visualised using a systems mapping approach, with data obtained from the Office for National Statistics and commissioners in the GM Health and Social Care Partnership.

Results Once vaccination data left primary care, it passed through 1 of 10 local child health information services (CHISs), using an assortment of different information technology systems, after which it shed individual identifiers and was aggregated within national systems. None of the existing GM CHISs were accessible to PED practitioners.

Conclusion More work needs to be done to explore possible alternative sources of accurate vaccination data during a PED consultation.

\section{INTRODUCTION}

Vaccination remains one of the great global public health successes. Since their discovery more than 300 years ago, vaccines have saved countless millions of lives, ${ }^{1}$ reduced the incidence of dozens of diseases and even led to the eradication of smallpox. ${ }^{2}$ However, in the UK, uptake of routine childhood vaccinations (provided by the National Health Service (NHS) at no cost to the parent/carer) has fluctuated over recent years and remains below WHO targets for a number of vaccinations (eg, the measles, mumps and rubella (MMR) vaccine). ${ }^{3}$ This finding is on a background of global changes in the pattern of vaccination and an associated increase in outbreaks of vaccine-preventable diseases, further compounded by disruptions to delivery of routine vaccination programmes during the SARS-CoV-2/COVID-19 pandemic.
Every year in England, millions of children and young people (CYP) attend the paediatric emergency department (PED) ${ }^{4}$ and may sometimes have a long wait to see a healthcare professional. In addition to their primary reason for presentation, CYP attending the hospital may have unmet health need (eg, sexual health) or may not be able to access preventive elements of routine healthcare (eg, vaccination) for a myriad of reasons. A hospital attendance might therefore be an opportunity to improve health, beyond the initial reason for presentation, and early work has shown that this would be an acceptable approach to parents/carers. ${ }^{5}$

If any child or young person who have not had their age-appropriate vaccinations is identified during a PED attendance, clinicians may (should it be clinically/situationally appropriate) be able to offer one or more tailored interventions to address this. ${ }^{6}$ The benefits of such an approach are numerous and include ensuring appropriate management, for example, in the case of a tetanusprone wound (where management depends on vaccination status), and increasing community coverage in case of an outbreak of a vaccine-preventable disease, for example, measles.

However, in order to be able to intervene with those at greatest risk of being undervaccinated, it is first necessary to be able to identify them in a timely and accurate way, given the time-limited interaction in the department and departmental pressures. Guidance recommends that professionals 'Check the immunisation status of CYP at every appropriate opportunity'. ${ }^{7}$ In the PED, therefore, all practitioners should routinely enquire of parents/carers accompanying a child or young person if they have had all their age-appropriate vaccinations. However, past work has shown that often no question 
is asked or recorded in the notes, ${ }^{8}$ and if the enquiry is made, it is usually done in a superficial way via a question such as 'Have they had all their vaccinations?'. When asked, parents/carers tended to overestimate vaccination coverage. $^{5}$

In contrast, in primary care, if a child attends a general practitioner (GP) appointment, the clinician is alerted, via the presence of a 'pop-up', if the child is not up to date with his or her vaccinations. The difference here is that the vaccination data are held within the same system as the GP records, but the hospital systems are separate. In the UK, the majority of routine childhood immunisations are offered in community locations, commonly delivered via settings such as a GP surgery. Administration of one or more vaccines will be recorded in the GP electronic system, with returns sent from these systems to the local child health information service (CHIS) and then on to the central surveillance system.

The objective of this work was to map the CHIS network in Greater Manchester (GM) to assess its potential as a source of accurate vaccination data for clinicians working in PEDs across the region, given the issues with obtaining information from parents/carer. This work was carried out as part of a bigger project looking at the potential for a PED-delivered vaccination intervention.

\section{METHODS}

The work was carried out in GM, England. The Office for National Statistics (ONS) mid-2019 estimates were used to describe the GM population of CYP $<16$ years old. ${ }^{9}$ Names of local authorities (LAs) and associated CHISs, the provider organisations for each CHIS and the data management systems used were obtained via requests to GM Health and Social Care Partnership (GMHSCP), the organisation responsible for commissioning vaccination services in GM. Lists of LAs, CHIS and provider organisations (where relevant) were compiled and then combined with ONS data using systems mapping, ${ }^{10}$ an approach commonly used in public health. The map in figure 1 (which represents the structure of the system in GM in mid-2020) was created using Microsoft Visio V.2016 and fact-checked by GMHSCP before the names of individual organisations and information technology (IT) systems were removed (to protect commercially sensitive information).

\section{RESULTS}

In GM, a population of around 582000 CYP had their vaccination data held by 10 different CHISs, provided by four different organisations, using three different national IT management systems commissioned in GM (although this has recently been reduced to two). Figure 1 shows the population served (by LA), the CHIS holding and managing data for each population, and the provider organisations commissioned to manage multiple CHISs (where relevant). Flow of vaccination data is represented by directional arrows (labelled with the IT system used).

No CHIS was accessible to practitioners working in secondary care (each system is password protected and only accessible to those working in community-based services), nor was there a focal point for GM that would have acted as a meaningful target for connecting the CHISs to secondary care data systems (aside from issues of interoperability) as none of the CHISs were connected to each other (even if managed by the same provider organisation). Once the vaccination data left GM CHISs, they shed individual identifiers and progressed up the national system in an aggregated anonymised format.

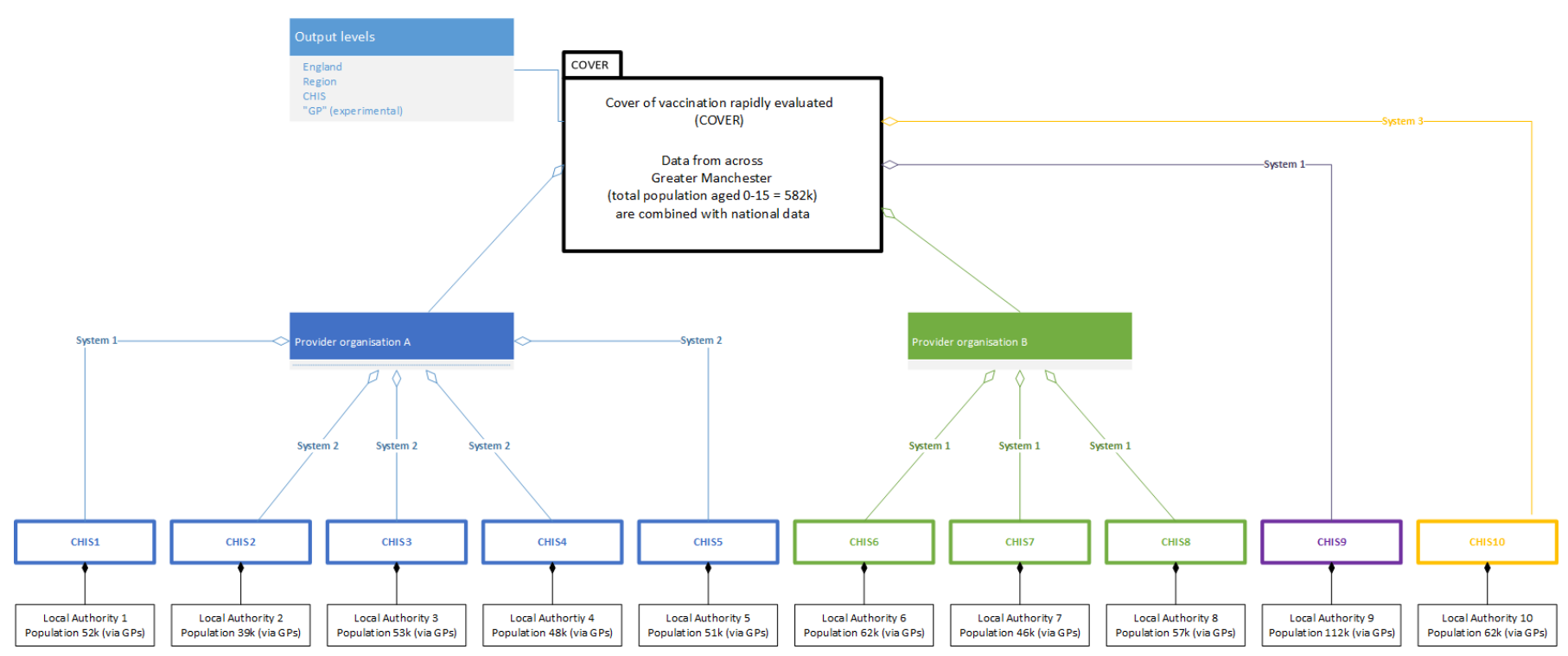

Figure 1 Management of data relating to vaccination in children and young people (aged $<16$ years old) in Greater Manchester. The names of the local providers and systems have been anonymised. Population=Office for National Statistics 2019 mid-year estimate for those aged 0-15 years inclusive to the nearest 1000. CHIS, child health information service; GP, general practitioner. 


\section{DISCUSSION}

CYP attending settings such as the PED may benefit from interventions to improve vaccination coverage; however, it is not currently possible to reliably identify those who are not up to date. Although parent/carer recall remains the most common source of vaccination data during a PED consultation, clinicians often do not take a (meaningful) vaccination history and parent/carer recall tends towards overestimation. ${ }^{5} 811$ An alternative approach is needed for checking vaccination status for all CYP as part of routine care but would also add value in special circumstances, such as those where subsequent medical management might be altered by the child's vaccination status (eg, tetanus) or in controlling outbreaks of vaccinepreventable diseases (eg, measles).

Potential alternative sources of data include the Red Book (a handheld paper or electronic record of child health), the GP summary care record (where available and accessible), phoning GP surgeries (on an individual patient basis) and local CHIS. This work has used a system mapping to approach to show that, while an individual CHIS may contain accurate vaccination data, it is inaccessible to hospital-based clinicians and also part of a prohibitively complex system with no single focal point, so it does not represent a viable option in GM at the current time. The simplest solution might be a unified regional CHIS, but that is a commissioning decision beyond the influence of secondary care clinicians. A limitation of the study is that it used only a single mapping approach to visualise the data. Another potential limitation is that GM has a commissioning structure which may not be replicated elsewhere, so collating the CHIS data may be more complex in other settings.

Future work will look at the potential for accessing primary care-held vaccination data (eg, via summary care records) as an alternative. However, preliminary work suggests that while these records are technically accessible, extracting relevant data takes a disproportionate amount of time as the vaccination data are unstructured and only interpretable by someone with an extensive working knowledge of the NHS childhood vaccination schedule.

Until a viable (in terms of time and effort for clinicians), accurate and real-time alternative to parent/carer recall is available, it is not going to be possible to progress to delivering an intervention to those CYP who are undervaccinated at the time of their attendance to the PED.

\section{CONCLUSIONS}

The PED offers an underused opportunity to deliver interventions to improve the wider health and well-being of patients, with vaccination being an example of such an intervention. However, the lack of access to reliable vaccination data in a timely fashion, during a PED attendance, means that it is not currently possible to identify those CYP in need of an intervention. The complex structures of postprimary care data management mean that in
GM, the CHISs, while considered the definitive source of vaccine data, are wholly inaccessible in their current form and are therefore not a viable source of vaccination information for clinicians working in the PED.

Twitter Rachel Isba @dr_asbo

Acknowledgements Thanks to colleagues on the Great Manchester Screening and Immunisation Team for their invaluable input into the system mapping work.

Contributors Rl conceived of the study, collected and mapped the data, and was involved in all stages of preparation of this manuscript. JK and ND were involved in all stages of the design of the study and in writing and revising the manuscript.

Funding The authors have not declared a specific grant for this research from any funding agency in the public, commercial or not-for-profit sectors.

Competing interests None declared.

Patient consent for publication Not applicable.

Ethics approval This study does not involve human participants.

Provenance and peer review Not commissioned; externally peer reviewed.

Data availability statement Data sharing is not applicable as no datasets were generated and/or analysed for this study.

Open access This is an open access article distributed in accordance with the Creative Commons Attribution Non Commercial (CC BY-NC 4.0) license, which permits others to distribute, remix, adapt, build upon this work non-commercially, and license their derivative works on different terms, provided the original work is properly cited, appropriate credit is given, any changes made indicated, and the use is non-commercial. See: http://creativecommons.org/licenses/by-nc/4.0/.

\section{ORCID iDs}

Rachel Isba http://orcid.org/0000-0002-2896-4309

Jo Knight http://orcid.org/0000-0002-7148-1660

\section{REFERENCES}

1 Plotkin S. History of vaccination. Proc Natl Acad Sci U S A 2014;111:12283-7.

2 World Health Organization. Smallpox. Available: https://www.who.int/ westernpacific/health-topics/smallpox [Accessed 06 Sep 2021].

3 European Centre for Disease Prevention and Control. Vaccination coverage for the second dose of measles-containing vaccine, EU/ EEA, 2018. Available: https://www.ecdc.europa.eu/en/publicationsdata/vaccination-coverage-second-dose-measles-containingvaccine-eueea-2018 [Accessed 06 Sep 2021].

4 NHS Digita. Hospital Accident \& Emergency Activity 2019-20, 2021. Available: https://digital.nhs.uk/data-and-information/publications/ statistical/hospital-accident-emergency-activity/2019-20 [Accessed 06 Sep 2021].

5 Isba R, Edge R. Delivery of a multi-focus public health intervention in the paediatric emergency department: a feasibility and acceptability pilot study. BMJ Open 2021;11:e047139.

6 Edge R, Isba R. Interventions delivered in secondary or tertiary medical care settings to improve routine vaccination uptake in children and young people: a scoping review protocol. JBI Evid Synth 2020;18:1566-72.

7 National Institute for Health and Care Excellence. Immunisations: reducing differences in uptake in under 19, 2021. Available: https:// www.nice.org.uk/guidance/ph21 [Accessed 06 Sep 2021].

8 Newell K, Rousseva C, Slade C, et al. Should we offer opportunistic vaccination in the paediatric emergency department? Emerg Med $\mathrm{J}$ 2015;32:1007.2-8.

9 Office for National Statistics. Population estimates for the UK, England and Wales, Scotland and Northern Ireland: mid-2019, 2020. Available: https://www.ons.gov.uk/releases/populationestimatesforth eukenglandandwalesscotlandandnorthernirelandmid2019 [Accessed 06 Sep 2021].

10 Egan M, McGill E, Penney T. NIHR SPHR guidance on systems approaches to local public health evaluation. Part 1: introducing systems thinking. London: National Institute for Health Research Scholl for Public Health Research, 2019. https://sphr.nihr.ac.uk/wpcontent/uploads/2018/08/NIHR-SPHR-SYSTEM-GUIDANCE-PART1-FINAL_SBnavy.pdf

11 Conway SP. Opportunistic immunisation in hospital. Arch Dis Child 1999;81:422-5. 